\title{
A case-control genome-wide association study of ADHD discovers a novel association with the tenascin $\mathrm{R}(T N R)$ gene
}

\author{
Ziarih Hawi ${ }^{1}$, Hannah Yates ${ }^{1}$, Ari Pinar $\mathbb{B}^{1}$, Aurina Arnatkeviciute ${ }^{1}$, Beth Johnson ${ }^{1}$, Janette Tong $\mathbb{B}^{1}$, Kealan Pugsley ${ }^{1}$, \\ Callum Dark', Marc Pauper', Marieke Klein $\mathbb{D}^{2}$, Helen S. Heussler ${ }^{3}$, Harriet Hiscock', Alex Fornito', Jeggan Tiego', \\ Amy Finlay ${ }^{1}$, Alasdair Vance ${ }^{5}$, Michael Gill $\mathbb{D}^{6}$, Lindsey Kent ${ }^{7}$ and Mark A. Bellgrove ${ }^{1}$
}

\begin{abstract}
It is well-established that there is a strong genetic contribution to the aetiology of attention deficit hyperactivity disorder (ADHD). Here, we employed a hypothesis-free genome-wide association study (GWAS) design in a sample of 480 clinical childhood ADHD cases and 1208 controls to search for novel genetic risk loci for ADHD. DNA was genotyped using Illumina's Human Infinium PsychArray-24v1.2., and the data were subsequently imputed to the 1000 Genomes reference panel. Rigorous quality control and pruning of genotypes at both individual subject and single nucleotide polymorphism (SNP) levels was performed. Polygenic risk score (PGRS) analysis revealed that ADHD case-control status was explained by genetic risk for ADHD, but no other major psychiatric disorders. Logistic regression analysis was performed genome-wide to test the association between SNPs and ADHD case-control status. We observed a genome-wide significant association ( $p=3.15 \mathrm{E}-08)$ between ADHD and rs6686722, mapped to the Tenascin R (TNR) gene. Members of this gene family are extracellular matrix glycoproteins that play a role in neural cell adhesion and neurite outgrowth. Suggestive evidence of associations with ADHD was observed for an additional 111 SNPs ( $\leqslant 9.91 \mathrm{E}-05)$. Although intriguing, the association between DNA variation in the TNR gene and ADHD should be viewed as preliminary given the small sample size of this discovery dataset.
\end{abstract}

\section{Introduction}

Attention deficit hyperactivity disorder (ADHD) is the most commonly diagnosed neurodevelopmental disorder of childhood. It is typically characterised by a persistent pattern of inattention, impulsivity and/or hyperactivity. Longitudinal studies indicate that approximately $66-77 \%$ of individuals who experience childhood ADHD continue to experience at least subthreshold symptoms of ADHD that significantly impact adulthood functioning ${ }^{1,2}$. The global prevalence of $\mathrm{ADHD}$ has been estimated at

\footnotetext{
Correspondence: Ziarih Hawi (ziarih.hawi@monash.edu)

${ }^{1}$ School of Psychological Sciences and Monash Institute for Cognitive and Clinical Neurosciences (MICCN), Monash University, Melbourne, Australia

${ }^{2}$ Departments of Human Genetics, and Psychiatry, Radboud University Medical Center, Nijmegen, The Netherlands
}

Full list of author information is available at the end of the article.
$5.2-7.2 \%^{3,4}$. Although the aetiology of ADHD is not well defined, genetic and environmental factors have been implicated in the disorder. Despite minor disparities across individual samples and study designs, the overall heritability of ADHD is estimated at $70-90 \% \%^{5,6}$.

The efficiency of indirect dopamine agonists in reducing the symptoms of ADHD led to the development of the 'dopamine hypothesis of ADHD', which postulated that dysregulated dopamine signalling is central to the pathophysiology of $\mathrm{ADHD}^{7}$. Putative disruption of other monoamines such as noradrenaline and serotonin in ADHD has also been proposed. Accordingly, genetic markers mapped to these monoamine pathways have historically been pursued as candidate genes for ADHD. Although a number of replicated findings exist within this candidate gene literature ${ }^{8,9}$, a key limitation remains the a 
priori selection of genes based upon incomplete knowledge of the biology of ADHD, which may ultimately impede the identification of novel risk markers.

Genome-wide association studies (GWAS) allow the identification of novel risk variants without prior knowledge of the biology of a trait or disorder. Further, this approach is aligned with the polygenic nature of complex disorders whereby the small role of individual single nucleotide polymorphisms (SNPs) can be considered in aggregate to better understand how genetic susceptibility may arise. To date, 13 ADHD-GWAS have been published. Of these, seven were case-control studies ${ }^{10-16}$, two were family-based analyses ${ }^{17,18}$, and three examined the association between quantitative ADHD symptom measures and genetic markers ${ }^{19-21}$. A GWAS meta-analysis was also performed in $2010^{22}$. Overall, these previous ADHD-GWAS had limited success in identifying associations. However, preliminary evidence of associations (albeit below GWAS significance) were identified for genes that function in biological processes relevant to ADHD aetiology. For example, pathway analysis highlights a potential role for potassium channel genes and activation Ras Homologue Family Member A (RhoA) signalling genes, lending further support to hypothesised dysregulation of neurotransmitter release in $\mathrm{ADHD}^{23}$. Additional pathway analysis utilising data arising from five ADHD-GWAS using the Ingenuity and BiNGO tools, showed significant enrichment of genes mapped to a network involved in neurite outgrowth whose targets are modulated by drugs used to treat $\mathrm{ADHD}^{24}$. Most recently, a large meta-analysis of GWAS data arising from 20,183 ADHD cases and 35,191 controls yielded the first 12 independently significant ADHD-GWAS loci ${ }^{25}$.

Here, we conducted a GWAS on a rigorously diagnosed clinical ADHD cohort collected across Australia, England and Ireland in an attempt to clarify further the genetic architecture of ADHD and to potentially identify novel genetic risk factor(s). An additional important purpose of the current study was to contribute to the expansion of the international GWAS community. In this context, GWAS data derived from this study can be combined with those of the international ADHD-GWAS community (e.g., ADHD-PGC) for subsequent GWAS analyses.

\section{Materials and methods Participants}

Five hundred and sixty seven $(N=567)$ children with ADHD of European ancestry were recruited from Australia, the United Kingdom and Ireland. None of the ADHD probands were included in previously published GWAS. All cases met the DSM-IV diagnostic criteria for ADHD at the time of sample collection. ADHD status was determined through parental semi-structured interview and completion of the Conners' Parent Rating Scale for
ADHD, which has demonstrated internal reliability and criterion validity for use in assessing $\mathrm{ADHD}^{26}$. Children with an IQ less than 70 as determined using the Wechsler Intelligence Scale for Children version 4 (WISC-IV Standardisation Sample, 2003) ${ }^{27}$ were excluded. One thousand two hundred and ninety-six control $(N=1296)$ participants were also recruited. All patients and controls were European by descent based on self-report ethnicity of all four grandparents ${ }^{28}$. All control participants were recruited in Australia and had no self-reported personal history of psychiatric or neurological disorders including ADHD. Written informed consent was provided by the individual in the case of adults, or the primary caregiver or guardian in the case of children/adolescents.

\section{DNA genotyping and quality control}

DNA samples were genotyped using the Illumina Infinium PsychArray-24v1.2 BeadChip at Path West's Diagnostic Genomics Laboratory in Western Australia. The Illumina Psych-Chip has a backbone of 510,000 markers comprising 265,000 tagging SNPs found on the Infinium Core-24 BeadChip and 245,000 markers from the Infinium Exome-24 BeadChip. It was developed in collaboration with the Psychiatric Genomics Consortium (PGC) and supplemented with an additional $\sim 50,000$ SNPs implicated in psychiatric and neurodevelopmental disorders. To avoid spurious GWAS findings, we adopted a stringent quality control (QC) protocol using PLINK 1.9 software at both the individual subject and SNP level ${ }^{29,30}$.

\section{Subject-level QC}

The following subject-level QC was employed: (1) We initially removed individuals with low-genotyping score by excluding participants with $\geqslant 0.03$ of missing data; (2) we performed identity by descent analysis to detect and remove possible sample contaminations, duplications as well as unknown familial relationships (such as relatedness); (3) we applied principal components analysis to identify any potential sources of population stratification and removed outlier subjects; (4) we tested for unusual heterozygosity, which refers to the presence of more or less heterozygous SNPs across the genome than would be expected by the population mean, and removed individuals displaying outlying mean heterozygosity (greater than \pm 3 SDs from the sample mean); and (5) disparities between recorded and observed sex status were determined through X-chromosome homozygosity were removed.

Following the above rigorous QC, 480 ADHD probands (Australians $=365$, English $=62$ and Irish $=53$ ) remained in the final set for analysis. All children were aged between 5 and 18 years (age mean; Age $_{M}=10.27$ years, Age $_{\mathrm{SD}}=3.03$ ). Of the ADHD cases $87 \%$ were male and 
$13 \%$ were female. The high frequency of male participants in the ADHD cohort is reflective of the sex ratio in clinical populations $^{31}$. For controls, 1208 participants aged between 7 and 60 years $\left(\right.$ Age $_{\mathrm{M}}=20.61$ years, Age $\mathrm{SD}_{\mathrm{D}}=$ 6.76) were carried forward to the final statistical association analysis. Of these participants, $49 \%$ were male and $51 \%$ were female.

\section{SNP-level QC}

Prior to imputation, additional filtering steps were conducted by removing 3516 SNPs with genotyping call rate $<95$. In addition, genotyped SNPs departing from Hardy-Weinberg $(\mathrm{H}-\mathrm{W})$ equilibrium were also excluded. This resulted in the removal of 76 SNPs. A further 1855 SNPs with significantly different $(p \leq 1.0 \mathrm{E}-5)$ missing genotype rates between cases and controls were also removed. Finally, SNPs with a minor allele frequency (MAF) $<0.01$ were removed leaving 290,265 in the final set taken forward to imputation.

\section{DNA imputation}

The freely available software packages $\mathrm{MaCH}$ and Minimac2 were used for phasing and genotype imputation employing the 1000 Genomes reference panel (hg 19 build 37$)^{32,33}$. Finally, a MAF $\geqslant 0.05$ was implemented for our final association analysis. These constraints yielded a final set of 5,407,269 SNPs which were subjected to statistical association analysis.

\section{PGRS calculation}

Polygenic risk scores (PGRS) for the five major psychiatric disorders including ADHD, autism spectrum disorder (ASD), schizophrenia (SCZ), bipolar disorder (BD) and major depressive disorder (MDD) were calculated using PRSice software package ${ }^{34}$. PGRS for each subject and disorder were estimated as a sum of risk alleles weighted by their effect size as defined by data arising from the latest publically available GWAS ${ }^{25,35-38}$. For each disorder PGRS were calculated at a $1000 p$ thresholds $\left(p_{\mathrm{T}}\right)$ ranging from 0.0005 to 0.5 . To find the most predictive $p_{\mathrm{T}}$ logistic regression was applied at each threshold using ADHD status as a regressor and Nagelkerke's $R^{2}$ and the corresponding $p$ values were estimated. Analysis of PGRS here served to demonstrate that the current ADHD cohort replicated the published and publically available genetic risk profile for ADHD.

\section{ADHD case-control genome-wide association analysis}

Association analysis was performed in 480 ADHD cases and 1208 controls using logistic regression analysis implemented in PLINK 1.9. The following covariates were included: age, age ${ }^{2}$, age $\times$ gender, and the top five eigenvectors accounting for population stratification.

\section{Results}

\section{PGRS analysis}

Here, we utilised PGRS analysis to determine whether our ADHD cohort replicated the published ADHD genetic risk profile. Logistic regression was used to examine the relationship between PGRS for each of the 5 major psychiatric disorders and ADHD case-control status, for $1000 p_{\mathrm{T}}$ values ranging from 0.0005 to 0.5 . Age, gender, age ${ }^{2}$, age $\times$ gender, along with the top five eigenvectors accounting for population stratification were used as covariates. As expected, only ADHD PGRS was significantly predictive of ADHD case-control status (Nagelkerke's $R^{2}=0.03, p=7.6 \mathrm{E}-15$ at $p_{\mathrm{T}}=0.0785$ ). PGRS for the other four psychiatric disorders did not reach the recommended statistical significance threshold of $p=0.001$ at any $p$-threshold (Figure 1$)^{34}$. The best model fit values for the remaining four psychiatric disorders were as follows: BP (Nagelkerke's $R^{2}=0.00285$, $p=0.012, p_{\mathrm{T}}=0.011$ ), MDD (Nagelkerke's $R^{2}=0.00250$, $p=0.018, p_{\mathrm{T}}=0.103$ ), ASD (Nagelkerke's $R^{2}=0.00125$, $p=0.093, p_{\mathrm{T}}=0.1$ ), SCZ (Nagelkerke's $R^{2}=0.00082, p=$ $\left.0.175, p_{\mathrm{T}}=0.0335\right)$.

\section{ADHD case-control genome-wide association analysis}

The Q-Q plot for the association analysis in $480 \mathrm{ADHD}$ cases and 1208 controls showed a slight inflation of $p$ values $(\lambda=1.08)$ relative to expectation under the null distribution (Fig. 2). This lies within acceptable limits $(\lambda=1.01-1.11)$ and the distribution is skewed at the extreme tail of low $p$ values, as expected. As can be seen from the Manhattan plot (Fig. 3) and Table 1, a significant association between ADHD and rs6686722, mapped $22.8 \mathrm{kbp}$ upstream of Tenascin $\mathrm{R}$ gene (TNR), was observed $(p=3.15 \mathrm{E}-08)$. The imputation quality of

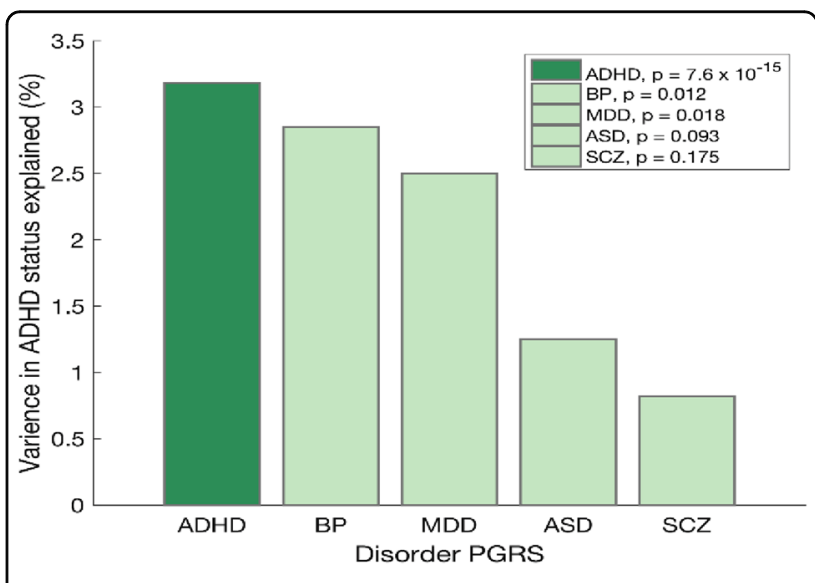

Fig. 1 Diagrammatic representation showing the polygenic risk scores (PGRS) for ADHD, BP, MDD, ASD and SCZ against the ADHD case-control statues for the current cohort. Only ADHD-PGRS were significantly predictive of ADHD status $(p=7.6 \times$ $10^{-15}$ ) explaining $3.25 \%$ of variance in the ADHD case-control status 
rs6686722 is very high $\left(r^{2}=0.98\right)$. Interestingly, and as presented in the regional association plot (Fig. 4), ten SNPs within this region were either significantly associated with ADHD or showed a strong trend towards statistical association, with $p$ values $\leqslant 3.48 \mathrm{E}-07$.

Three other genomic loci also showed strong trends towards association ( $p$ values ranging from $9.647 \mathrm{E}-07$ to 4.06E-07; Table 1). The first of these is rs2410116 ( $p=$ 4.06E-07), which is mapped into a gene desert region (Fig. 4) $300.2 \mathrm{kbp}$ upstream of the gene encoding Rho GTPase Activating Protein (also known as deleted in liver cancer; DLC1). The second SNP is $\operatorname{rs61975260}(p=$ $5.972 \mathrm{E}-07$ ), which is mapped to the spermatogenesis associated 7 gene (SPATA7). This suggestive association signal is located within a subregion of Chr14 (88788507-89355721) that comprises several genes

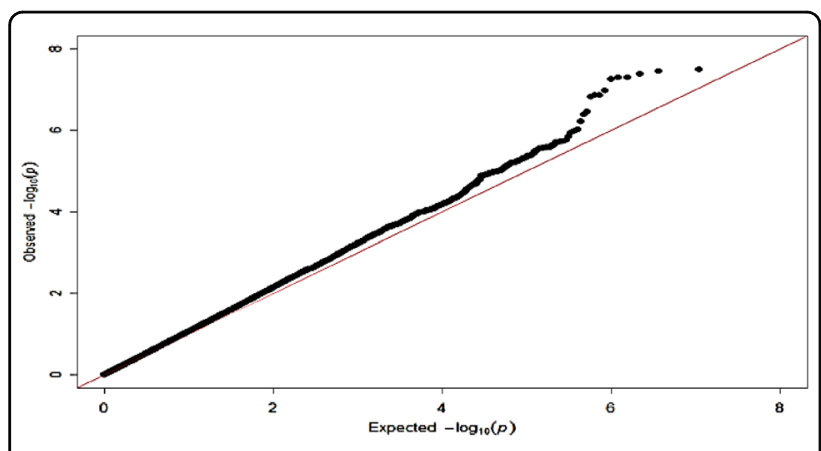

Fig. 2 Q-Q plot of ADHD-GWAS using 5,407,269 imputed SNPs A deviation in the observed $p$-value at the top end consistent with genetic influence including SPATA7, ZC3H14, PTPN21, EML5 and TTC8. Some of these genes, such as SPATA7 have been implicated in psychiatric conditions, including schizophrenia.

The third of these SNPs is rs77224013 ( $p=9.647 \mathrm{E}-07)$ which is mapped $58.4 \mathrm{kbp}$ upstream of the Interferon Alpha and Beta Receptor Subunit 2 gene (IFNAR2). This region of the genome is enriched for genes that function in the immune system (Fig. 4). In addition to the above SNPs, 108 other genomic variants displayed suggestive evidence of association (Table 1 and Supplementary Table 1) with $p$ values $\leqslant 9.91 \mathrm{E}-05$ to $1.0 \mathrm{E}-06$. We also explored replication of our results within the publically available database of the PGC-iPSYCH meta-analysis ${ }^{25}$. Supplementary Table 1 lists the comparable $p$ value within the PGC-iPSYCH meta-analysis against SNPs with $p$ values $\leqslant 9.91 \mathrm{E}-05$ in the current dataset. Notably, our leading SNP rs6686722 tends towards a nominally significant association in the PGC-iPSCYH meta-analysis $(p=0.07)$. Further, five SNPs from the current study were nominally significant in the PGC-iPSYCH meta-analysis (Supplementary Table 1).

\section{Discussion}

Here, we report the results of a GWAS of 480 probands with childhood ADHD and 1208 controls. PGRS analysis revealed that our sample replicated the published genetic risk profile for ADHD. In fact, PGRS of ADHD, but not other major psychiatric disorders (ASD, SCZ, BP and MDD), explained $3.25 \%$ of variance in ADHD case-control status in our cohort $(p=7.6 \mathrm{E}-15)$. These data demonstrate that the current dataset is enriched for

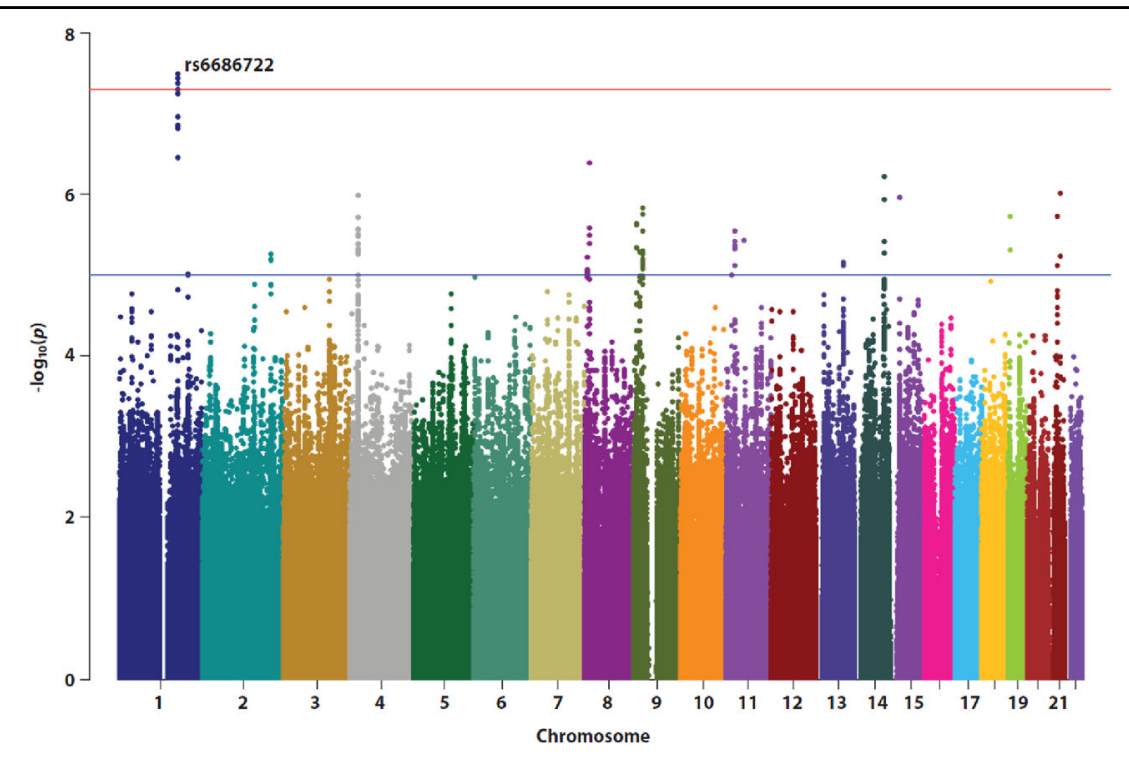

Fig. 3 Manhattan plot of the ADHD-GWAS showing $-10 \log (p$ value) versus genomic location for autosomal chromosomes (1-22). Results show a significant association of rs6686722 on chromosome $1(p=3.1 \mathrm{E}-08)$. The horizontal blue and orange lines represent $p$ values at $1.0 \times 1 \mathrm{E}-05$ and $5.0 \mathrm{E}-08$, respectively 
Table 1 Results of the ADHD case-control genome-wide statistical association analysis for the top 20 SNPs

\begin{tabular}{|c|c|c|c|c|c|c|c|c|}
\hline Chr & SNP & BP & $A 1^{*}$ & OR & L95 & U95 & $p$ Value & Nearest gene \\
\hline 1 & rs6686722 & 175733963 & T & 0.4167 & 0.3056 & 0.5682 & $3.15 \mathrm{E}-08$ & $22.8 \mathrm{kbp}$ upstream of TNR \\
\hline 8 & rs2410116 & 13673447 & A & 0.4991 & 0.3814 & 0.6531 & $4.06 \mathrm{E}-07$ & $300.2 \mathrm{kbp}$ upstream of DLC1 \\
\hline 14 & rs61975260 & 88895941 & G & 0.494 & 0.3745 & 0.6516 & 5.97E-07 & SPATA7 \\
\hline 21 & rs77224013 & 34543845 & A & 3.869 & 2.252 & 6.647 & $9.64 \mathrm{E}-07$ & $58.4 \mathrm{kbp}$ upstream of IFNAR2 \\
\hline 4 & rs28612433 & 25264373 & $\mathrm{~T}$ & 0.5385 & 0.4202 & 0.6902 & $1.01 \mathrm{E}-06$ & $\mathrm{P} \mid 4 \mathrm{~K} 2 \mathrm{~B}$ \\
\hline 15 & rs4778174 & 27969566 & A & 0.5353 & 0.4164 & 0.6881 & $1.07 \mathrm{E}-06$ & $30.5 \mathrm{kbp}$ downstream of OCA2 \\
\hline 19 & rs35624673 & 8134616 & $\mathrm{~T}$ & 0.5497 & 0.4299 & 0.7029 & $1.83 \mathrm{E}-06$ & FBN3 \\
\hline 21 & rs2015560 & 26028890 & G & 0.3596 & 0.2362 & 0.5475 & $1.86 \mathrm{E}-06$ & - \\
\hline 11 & rs10767556 & 26623713 & G & 2.029 & 1.509 & 2.728 & $2.80 \mathrm{E}-06$ & ANO3 \\
\hline 11 & rs28609353 & 55651658 & C & 0.4698 & 0.3411 & 0.6471 & $3.74 \mathrm{E}-06$ & TRIM51 \\
\hline 2 & rs4673294 & 205189083 & G & 0.5462 & 0.4209 & 0.7089 & $5.43 \mathrm{E}-06$ & $221.5 \mathrm{kbp}$ upstream of PARD3B \\
\hline 21 & rs112686226 & 34527379 & G & 2.903 & 1.831 & 4.602 & $5.78 \mathrm{E}-06$ & $74.9 \mathrm{kbp}$ upstream of IFNAR2 \\
\hline 8 & rs13439086 & 8374246 & C & 2.162 & 1.549 & 3.018 & $5.91 \mathrm{E}-06$ & $198.9 \mathrm{kbp}$ upstream of SGK223 \\
\hline 13 & rs9545903 & 82446913 & C & 1.747 & 1.37 & 2.228 & $6.91 \mathrm{E}-06$ & - \\
\hline 1 & rs1172198 & 205662718 & A & 1.699 & 1.344 & 2.149 & $9.53 \mathrm{E}-06$ & SLC45A3/NUCKS1 \\
\hline 9 & rs7035982 & 27417407 & A & 1.757 & 1.368 & 2.257 & $1.01 \mathrm{E}-05$ & MOB3B \\
\hline 9 & rs35289513 & 18263813 & G & 3.038 & 1.854 & 4.978 & $1.03 \mathrm{E}-05$ & $210.3 \mathrm{kbp}$ upstream of ADAMTSL1 \\
\hline 6 & rs4615440 & 963496 & G & 1.781 & 1.378 & 2.302 & $1.05 \mathrm{E}-05$ & LOC285768 \\
\hline 3 & rs938524 & 136521208 & G & 0.5791 & 0.4538 & 0.739 & $1.13 \mathrm{E}-05$ & SLC35G2/STAG1 \\
\hline 18 & rs2733140 & 28363540 & $\mathrm{~T}$ & 0.5769 & 0.4511 & 0.7378 & $1.18 \mathrm{E}-05$ & $250.2 \mathrm{kbp}$ downstream DSC3 \\
\hline
\end{tabular}

Chr chromosome, BP base pair position, * Allele 1, OR dds ratio, L95 lower confidence intervals, U95 upper confidence intervals, - gene desert region,

genetic risk for ADHD and thus makes a worthwhile contribution to the international GWAS effort.

Genome-wide association analysis further revealed a significant association with rs6686722 that survived the stringent GWAS correction for multiple comparisons $(p=3.15 \mathrm{E}-08)$. This SNP is located $22.8 \mathrm{kbp}$ upstream of the Tenascin $\mathrm{R}(T N R)$ gene. The TNR gene is a member of the Tenascin family of the neural extracellular matrix glycoproteins and is highly expressed in the central nervous system $^{39}$. TNR is known to function in biological processes such as neural cell adhesion, neurite outgrowth and modulation of sodium channel function ${ }^{39,40}$ that have been implicated in the aetiology of psychiatric disorders. In addition, TNR interacts with Fibronectin 1 (FN1), the latter being involved in cell adhesion and migration processes including embryogenesis. This interaction may modulate the adhesive properties of TNR during synapse maintenance, a process that is suggested as a risk mechanism for complex psychiatric disorders ${ }^{41}$. Moreover, genetic association studies have implicated the genomic region where TNR is located in several brain disorders including schizophrenia, Alzheimer's disease, narcolepsy and neurological sleep disorder ${ }^{42}$. Further, an additional SNP (rs875326) mapped $2.5 \mathrm{kbp}$ at the $3^{\prime}$ untranslated region of the TNR was reported to marginally associate with drug response in schizophrenia ${ }^{43}$. Although indirect, our TNR ADHD-GWAS finding, combined with findings reported for other psychiatric disorders, provides tentative support for a role of TNR in the aetiology of psychiatric conditions.

The second top SNP in our ADHD-GWAS analysis is mapped $300.2 \mathrm{kbp}$ upstream of the Rho GTPase Activating Protein (also known as DLC1). Recent studies have revealed common genetic susceptibilities to ADHD and smoking behaviour ${ }^{44}$. Adults with ADHD have higher rates of substance abuse, including higher rates of tobacco smoking ${ }^{44}$. In this context, a recent GWAS analysis of nicotine dependence reported genome-wide significant association with rs289519 (mapped to $D L C 1)^{45}$. Analysis of rare CNVs across two independent studies of $\mathrm{ASD}^{46,47}$, identified rare exonic loss within $D L C 1$ as risk variants for the disorder. Specifically, Prasad et al. ${ }^{46}$ identified one ASD individual who possessed a rare CNV deletion of $22 \mathrm{kbp}$ across a non-specified exonic region of DLC1. Furthermore, Woodbury-Smith et al. ${ }^{47}$ identified a $25 \mathrm{kbp}$ deletion encompassing exon nine on DLC1 in two 


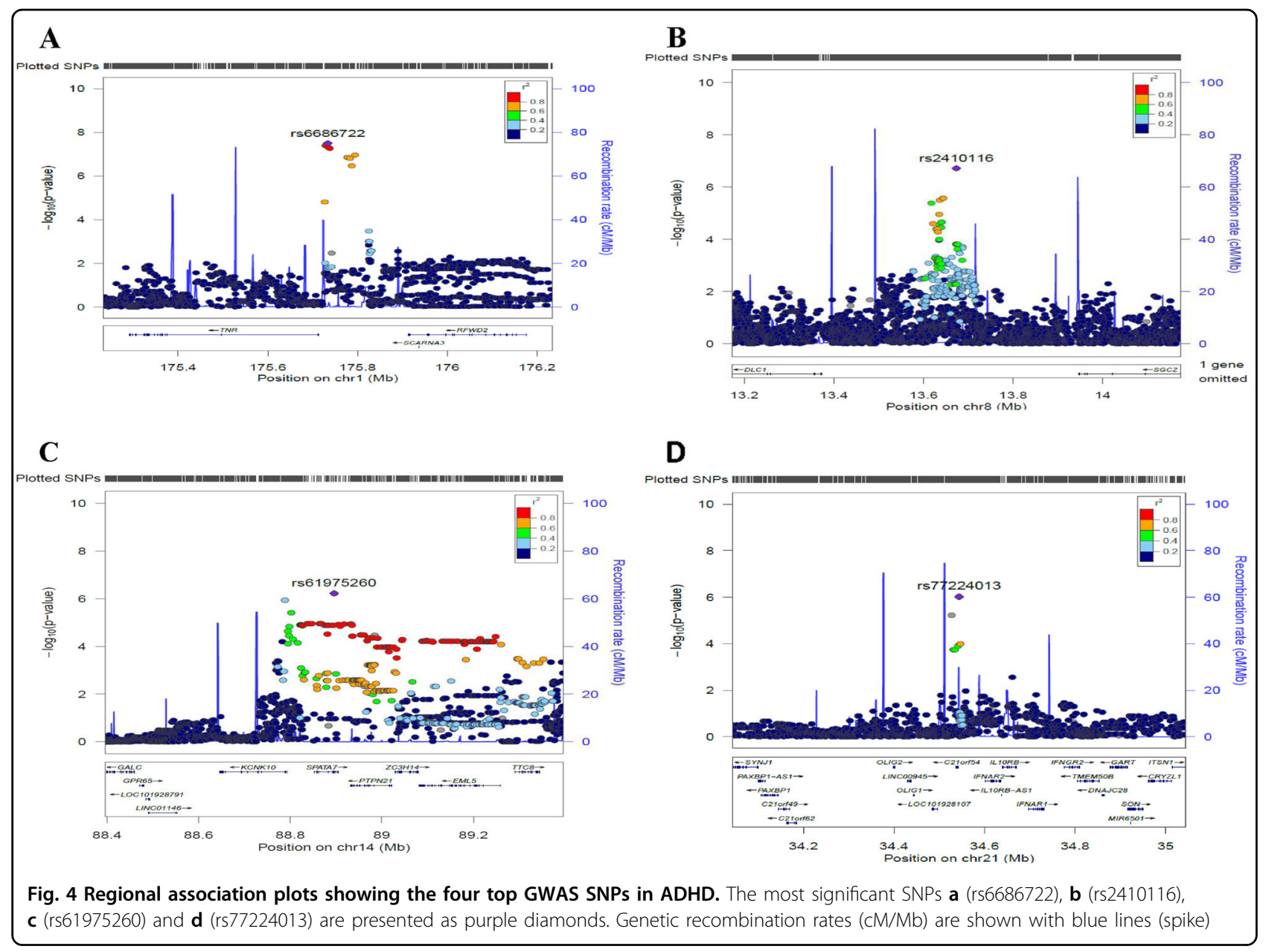

unrelated ASD-affected individuals. The above lines of evidence suggest that $D L C 1$ is a candidate gene worthy of further investigation as a potential susceptibility locus for ADHD and its comorbid disorders (either ASD or substance abuse).

An additional subthreshold association with ADHD was observed for rs61975260 $(p=5.972 \mathrm{E}-07)$ of the spermatogenesis associated 7 (SPATA7) gene which is expressed in the retina and cerebellum. This gene functions in the localisation of retinitis pigmentosa GTPase regulator interacting protein 1 to the photoreceptor connecting cilium (CC), as well as protein trafficking across the CC. Processing speed is a cognitive function that is compromised in psychiatric conditions including $\mathrm{ADHD}^{48,49}$. In this context, GWAS analysis ${ }^{49}$ of information processing speed measured using digit symbol, simple reaction time (RT), and 2 and 4-choice RT showed suggestive evidence of association between DNA variation in SPATA7 and 2-choice RT ( $p=2.71 \mathrm{E}-06)$.

Finally, suggestive evidence of association between ADHD and rs77224013 (9.65E-07) was also observed. Rs77224013 is located 58.4 and $94.8 \mathrm{kbp}$ upstream of the immune cytokine receptors interferon alpha and beta receptor subunit 2 (IFNAR2) and interleukin 10 receptor subunit beta (IL1ORB), respectively. Other immune modulator genes such as IL10RB-As1 and IFNAR1 also map to this region. Immune imbalance has been suggested as a predisposing factor for ADHD in genetically susceptible individuals ${ }^{50}$. Significantly increased transmission of IL-1Ra 4-repeat allele and decreased transmission of 2-repeat allele of a variable number tandem repeat polymorphism to ADHD-affected children was reported by Segman et al. ${ }^{51}$ Further, immune dysregulation is supported by the finding that ADHD individuals have four times higher concentrations of Interleukins ( $I L$ 1 and $I L-6)$ than typically developing children ${ }^{52}$. This led Verlaet et al. ${ }^{50}$ to hypothesise that "overproduction of these cytokines could lead to chronic inflammation in brain tissues". This is consistent with brain anomalies in children with ADHD. For example, Nopoulous et al. ${ }^{53}$ reported increased frequency of gray-matter heterotropia (ectopic nodules of neurons) and posterior fossa abnormalities in ADHD patients compared to controls. Further, reduced cortical volume associated with reduced 
surface area and gyrification were also reported in ADHD compared to controls ${ }^{54}$. However, the correlation between overproduction of cytokines and brain anomalies in ADHD requires further evidence to establish a causal link.

Our sample has provided replication evidence for some of the results arising from the recent and largest ADHD meta-analysis by the PGC-IPSYCH consortia ${ }^{25}$. For example, we report a nominal association with rs281324 $(p=0.045)$, mapped to intron 3 of semaphorin 6D (SEMA6D) gene which sits within $70 \mathrm{kbp}$ genomic region of significant LD. Members of the semaphorin gene family have been implicated as inhibitors or chemo-repellents in axon pathfinding and fasciculation and branching. More recently, Klein et al. ${ }^{55}$, examined if the genetic risk markers (reported by the PGC meta-analysis) mediate alteration in brain structure. They observed that rs 281323 (in perfect LD with rs281324) is significantly associated with increased risk for ADHD and putamen volume. Further, the SEMA6D rs281323 is strongly associated with the expression level of SEMA6D ${ }^{55}$. Together, these finding clearly implicate SEMA6D as a susceptibility locus for ADHD.

Finally, it is important to emphasise that our study has a number of major limitations. First, the discovery sample is small and has limited statistical power to detect a reliable genome-wide association signal. Further, our leading SNP association for TNR (rs6686722) is not significant within the much larger PGC-IPSYCH meta-analysis of ADHD $(p=0.07)$. Notwithstanding these limitations, our ADHD cohort is clearly enriched for genetic risk for ADHD, as evinced by our strong PGRS results for ADHD (but not the other major psychiatric disorders). As such, our study therefore makes an important contribution to the international genetics effort for ADHD.

\section{Acknowledgements}

This work has been supported by Project Grant funding from the National Health and Medical Research Council (NHMRC) of Australia to Z.H. (1006573, 1002458 and 1065677) and M.A.B. $(569636,1065677,1045354,1002458$ and 1006573).

\section{Author details \\ ${ }^{1}$ School of Psychological Sciences and Monash Institute for Cognitive and Clinical Neurosciences (MICCN), Monash University, Melbourne, Australia. ${ }^{2}$ Departments of Human Genetics, and Psychiatry, Radboud University Medical Center, Nijmegen, The Netherlands. ${ }^{3}$ Mater Research Institute, University of Queensland and Children's Health Queensland, South Brisbane, Australia. ${ }^{4}$ Pediatrics Royal Children's Hospital, Murdoch Children's Institute, Melbourne, Australia. ${ }^{5} T$ he Royal Children's Hospital, University of Melbourne, Victoria, Australia. ${ }^{6}$ Department of Psychiatry, Trinity College, Dublin, Ireland. ${ }^{7}$ School of Medicine, University of St Andrews, St. Andrews, Scotland, UK}

\section{Conflict of interest}

M.B. has received educational speaking fees from Shire within the last 5 years. Remaining authors declare that they have no conflict of interest.

\section{Publisher's note}

Springer Nature remains neutral with regard to jurisdictional claims in published maps and institutional affiliations.

Supplementary Information accompanies this paper at (https://doi.org/ 10.1038/s41398-018-0329-x).

Received: 23 April 2018 Accepted: 8 November 2018 Published online: 18 December 2018

\section{References}

1. Karam, R. G. et al. Persistence and remission of ADHD during adulthood: a 7year clinical follow-up study. Psychol. Med. 45, 2045-2056 (2015).

2. Uchida, M. Spencer, T. J. Faraone S. V. \& Biederman, J. Adult outcome of ADHD: an overview of results from the mgh longitudinal family studies of pediatrically and psychiatrically referred youth with and without ADHD of both sexes. J. Attten. Disord. 22, 523-534 (2018).

3. Polanczyk, G., de Lima, M. S., Horta, B. L., Biederman, J. \& Rohde, L. A. The worldwide prevalence of ADHD: a systematic review and metaregression analysis. Am. J. Psychiatry 164, 942-948 (2007).

4. Thomas, R., Sanders, S., Doust, J., Beller, E. \& Glasziou, P. Prevalence of attentiondeficit/hyperactivity disorder: a systematic review and meta-analysis. Pediatrics 135, e994-e1001 (2015).

5. Levy, F., Hay, D. A., McStephen, M., Wood, C. \& Waldman, I. Attention-deficit hyperactivity disorder: a category or a continuum? Genetic analysis of a largescale twin study. J. Am. Acad. Child Adolesc. Psychiatry 36, 737-744 (1997).

6. Larsson, H., Chang, Z., D'Onofrio, B. M. \& Lichtenstein, P. The heritability of clinically diagnosed attention deficit hyperactivity disorder across the lifespan. Psychol. Med. 44, 2223-2229 (2014).

7. Levy, F. The dopamine theory of attention deficit hyperactivity disorder (ADHD). Aust. N. Z. J. Psychiatry 25, 277-283 (1991).

8. Gizer, I. R., Ficks, C. \& Waldman, I. D. Candidate gene studies of ADHD: a metaanalytic review. Hum. Genet. 126, 51-90 (2009).

9. Hawi, Z. et al. The molecular genetic architecture of attention deficit hyperactivity disorder. Mol. Psychiatry 20, 289-297 (2015).

10. Lesch, K. P. et al. Molecular genetics of adult ADHD: converging evidence from genome-wide association and extended pedigree linkage studies. J. Neural Transm. 115, 1573-1585 (2008).

11. Neale, B. M. et al. Case-control genome-wide association study of attentiondeficit/hyperactivity disorder. J. Am. Acad. Child Adolesc. Psychiatry 49, 906-920 (2010).

12. Hinney, A. et al. Genome-wide association study in German patients with attention deficit/hyperactivity disorder. Am. J. Med. Genet. B Neuropsychiatr. Genet. 156B, 888-897 (2011).

13. Stergiakouli, E. et al. Investigating the contribution of common genetic variants to the risk and pathogenesis of ADHD. Am. J. Psychiatry 169, 186-194 (2012).

14. Yang, L. et al. Polygenic transmission and complex neuro developmental network for attention deficit hyperactivity disorder: genome-wide association study of both common and rare variants. Am. J. Med. Genet. B Neuropsychiatr. Genet. 162B, 419-430 (2013).

15. Zayats, T. et al. Genome-wide analysis of attention deficit hyperactivity disorder in Norway. PLoS ONE 10, e0122501 (2015).

16. Sanchez-Mora, C. et al. Case-control genome-wide association study of persistent attention-deficit hyperactivity disorder identifies FBXO33 as a novel susceptibility gene for the disorder. Neuropsychopharmacology 40, 915-926 (2015).

17. Neale, B. M. et al. Genome-wide association scan of attention deficit hyperactivity disorder. Am. J. Med Genet. B Neuropsychiatr. Genet. 147B, 1337-1344 (2008).

18. Mick, E. et al. Family-based genome-wide association scan of attention-deficit/ hyperactivity disorder. J. Am. Acad. Child Adolesc. Psychiatry 49, 898-905 (2010).

19. Lasky-Su, J. et al. Genome-wide association scan of quantitative traits for attention deficit hyperactivity disorder identifies novel associations and confirms candidate gene associations. Am. J. Med. Genet. B Neuropsychiatr. Genet. 147, 1345-1354 (2008).

20. Ebejer, J. L. et al. Genome-wide association study of inattention and hyperactivity-impulsivity measured as quantitative traits. Twin Res. Hum. Genet. 16, 560-574 (2013). 
21. Alemany, S. et al. New suggestive genetic loci and biological pathways for attention function in adult attention-deficit/hyperactivity disorder. Am. J. Med. Genet. B Neuropsychiatr. Genet. 168, 459-470 (2015).

22. Neale, B. M. et al. Meta-analysis of genome-wide association studies of attention-deficit/hyperactivity disorder. J. Am. Acad. Child Adolesc. Psychiatry 49, 884-897 (2010).

23. Mooney, M. A., McWeeney, S. K., Faraone, S. V., Hinney, A. \& Hebebrand, J. IMAGE2 Consortium et al. Pathway analysis in attention deficit hyperactivity disorder: an ensemble approach. Am. J. Med. Genet. B Neuropsychiatr. Genet. 171, 815-826 (2016)

24. Poelmans, G., Pauls, D. L., Buitelaar, J. K. \& Franke, B. Integrated genome-wide association study findings: identification of a neurodevelopmental network for attention deficit hyperactivity disorder. Am. J. Psychiatry 168, 365-377 (2011)

25. Demontis D., et al. Discovery of the first genome-wide significant risk loci for ADHD. bioRxiv 2017 https://doi.org/10.1101/145581.

26. Conners, C. K., Sitarenios, G., Parker, J. D. A. \& Epstein, J. N. The revised Conners' Parent Rating Scale (CPRS-R): factor structure, reliability, and criterion validity. J. Abnorm. Child Psychol. 26, 257-268 (1998).

27. WISC-IV Standardization Sample. WISC-IV Technical Report \# 2 Psychometric Properties 2003. Retrieved from: http://images.pearsonclinical.com/images/ pdf/wisciv/WISCIVTechReport2.pdf.

28. Rosenberg, N. A. et al. Genetic structure of human populations. Science 298, 2381-2385 (2002).

29. Anderson, C. A. et al. Data quality control in genetic case-control association studies. Nat. Protoc. 5, 1564-1573 (2010).

30. Turner S., et al. Quality control procedures for genome-wide association studies. Curr. Protoc. Hum. Genet. 19. https://doi.org/10.1002/0471142905. hg0119s68 Chapter 1, Unit 1 (2012).

31. Ford, T., Goodman, R. \& Meltzer, H. The British Child and Adolescent Mental Health Survey 1999: the prvalence of DSM-IV disorders. J. Am. Acad. Child Adolesc. Psychiatry 42, 1203-1211 (2003).

32. Howie, B., Fuchsberger, C., Stephens, M., Marchini, J. \& Abecasis, G. R. Fast and accurate genotype imputation in genome-wide association studies through pre-phasing. Nat. Genet. 44, 955-959 (2012).

33. Fuchsberger, C., Abecasis, G. R. \& Hinds, D. A. minimac2: faster genotype imputation. Bioinformatics 31, 782-784 (2015).

34. Euesden, J., Lewis, C. M. \& O'Reilly, P. F. PRSice: Polygenic Risk Score software. Autism Spectrum Disorders Working Group of the Psychiatric Genomics Consortium. Bioinformatics 31, 1466-1468 (2015).

35. Autism Spectrum Disorders Working Group of The Psychiatric Genomics Consortium. Meta-analysis of GWAS of over 16,000 individuals with autism spectrum disorder highlights a novel locus at 10q24.32 and a significant overlap with schizophrenia. Mol. Autism 8, 21 (2017).

36. Schizophrenia Working Group of the Psychiatric Genomics Consortium. Biological insights from 108 schizophrenia-associated genetic loci. Nature 511, 421-427 (2014)

37. Psychiatric GWAS Consortium Bipolar Disorder Working Group. Large-scale genome-wide association analysis of bipolar disorder identifies a new susceptibility locus near ODZ4. Nat. Genet. 43, 977-983 (2011).
38. Working Group of the Psychiatric GWAS Consortium, Ripke, S., Wray, N. R., Lewis, C. M., Hamilton, S. P. \& Weissman, M. M. Breen Gю A mega-analysis of genome-wide association studies for major depressive disorder. Major Depressive Disorderю. Mol. Psychiatry 18, 497-511 (2013).

39. Anlar, B. \& Gunel-Ozcan, A. Tenascin-R: role in the central nervous system. Int. J. Biochem. Cell. Biol. 44, 1385-1389 (2012).

40. $\mathrm{Xu}, \mathrm{J}$. C. et al. The extracellular matrix glycoprotein tenascin-R regulates neurogenesis during development and in the adult dentate gyrus of mice. J. Cell. Sci. 127, 641-652 (2014).

41. YC, Lin \& Koleske, A. J. Mechanisms of synapse and dendrite maintenance and their disruption in psychiatric and neurodegenerative disorders. Annu. Rev. Neurosci. I33, 349-378 (2010).

42. Zuo, L. et al. Genome-wide association study of alcohol dependence implicates KIAA0040 on chromosome 1q. Neuropsychopharmacology 37, 557-566 (2012).

43. Lavedan, C. et al. Association of the NPAS3 gene and five other loci with response to the antipsychotic iloperidone identified in a whole genome association study. Mol. Psychiatry 14, 804-819 (2009).

44. McClernon, F. J. \& Kollins, S. H.ADHD and Smoking: From Genes to Brain to Behavior. Ann. New Y. Acad. Sci. 1141, 131-147 (2008).

45. Gelernter, J. et al. Genome-wide association study of nicotine dependence in American populations: identification of novel risk loci in both AfricanAmericans and European-Americans. Biol. Psychiatry 77, 493-503 (2015).

46. Prasad, A. et al. A discovery resource of rare copy number variations in individuals with autism spectrum disorder. G3 (Bethesda) 2, 1665-1685 (2012).

47. Woodbury-Smith, M. et al. Using extended pedigrees to identify novel autism spectrum disorder (ASD) candidate genes. Hum. Genet. 134, 191-201 (2015).

48. Weiler, M. D., Bernstein, J. H., Bellinger, D. C. \& Waber, D. P. Processing speed in children with attention deficit/hyperactivity disorder, inattentive type. Child Neuropsychol. 6, 218-234 (2000).

49. Luciano, M. et al. Whole genome association scan for genetic polymorphisms influencing information processing speed. Biol. Psychol. 86, 193-202 (2011).

50. Verlaet, A. A., Noriega, D. B., Hermans, N. \& Savelkoul, H. F. Nutrition, immunological mechanisms and dietary immunomodulation in ADHD. Eur. Child Adolesc. Psychiatry 23, 519-529 (2014).

51. Segman, R. H. et al. Preferential transmission of interleukin-1 receptor antagonist alleles in attention deficit hyperactivity disorder. Mol. Psychiatry 7, 72-74 (2002).

52. Buske-Kirschbaum, A. et al. Psychoendocrine and psychoneuroimmunological mechanisms in the comorbidity of atopic eczema and attention deficit/ hyperactivity disorder. Psychoneuroendocrinology 38, 12-23 (2013).

53. Nopoulos, P. et al. Developmental brain anomalies in children with attentiondeficit hyperactivity disorder. J. Child Neurol. 15, 102-108 (2000).

54. Ambrosino, S., de Zeeuw, P., Wierenga, L. M., van Dijk, S. \& Durston, S. What can cortical development in attention-deficit/hyperactivity disorder teach us about the early developmental mechanisms involved? Cereb. Cortex 27, 4624-4634 (2017).

55. Klein M., et al. Genetic markers of ADHD-related variations in intracranial volume. BioRxiv 2017. https://doi.org/10.1101/184192. 